\title{
Dissémination et germination de semences issues des fèces de bétail au Sahel du Burkina Faso
}

\section{Pauline OUÉDRAOGO ${ }^{1}$ \\ Salifou TRAORÉ ${ }^{1}$ \\ Blandine Marie Ivette NACOULMA ${ }^{1}$ \\ Édith DABOUE ${ }^{2}$ \\ Babou André BATIONO 3}

${ }^{1}$ Université Joseph Ki-Zerbo

Unité de formation et de recherche en Sciences de la Vie et de la Terre

(UFR SVT)

03 BP 7021

Ouagadougou

Burkina Faso

${ }^{2}$ Centre national des semences forestières (CNSF)

01 BP 2682

Ouagadougou

Burkina Faso

${ }^{3}$ Institut de l'environnement et de recherches agricoles (INERA) Département Environnement et Forêts 01 BP 476

Ouagadougou

Burkina Faso

\section{Auteur correspondant /}

Corresponding author:

Pauline Ouédraogo -

paulineouedraogo@gmail.com

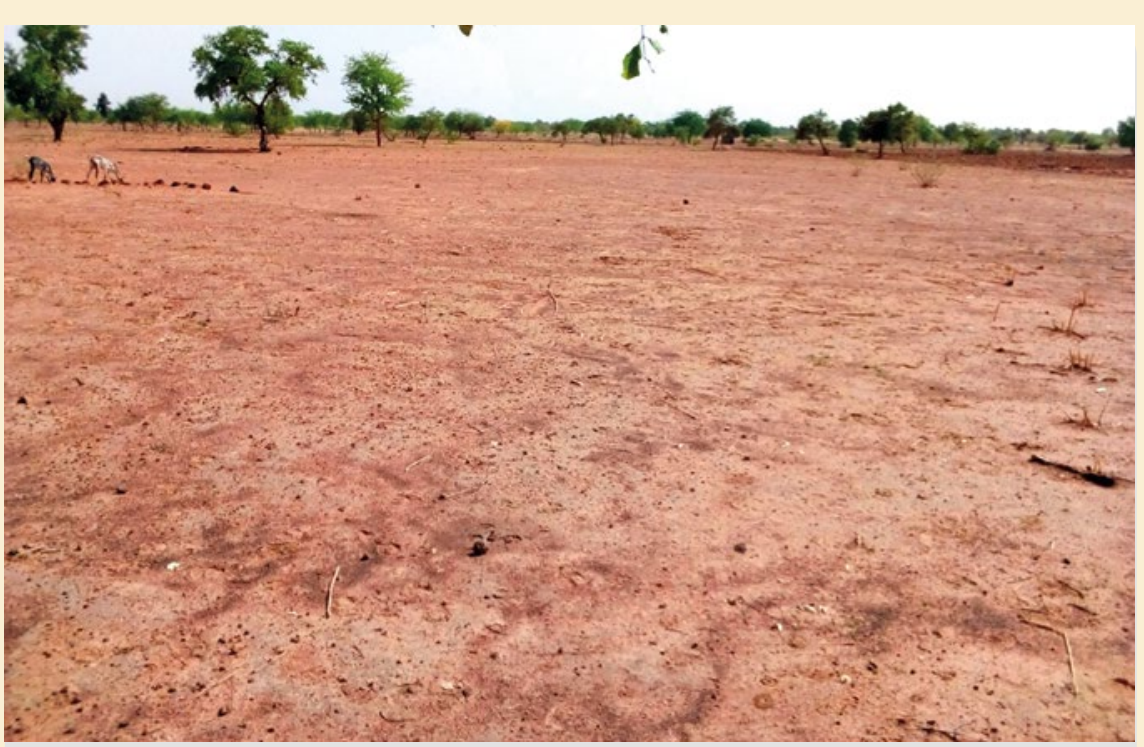

Photo 1.

Agroécosystème type de la zone d'étude. Typical agro-ecosystem of the study area. Photo P. Ouédraogo.

Doi : $10.19182 / \mathrm{bft} 2021.350 . a 36826$ - Droit d’auteur (c) 2021, Bois et Forêts des Tropiques - (c) Cirad - Date de soumission : 21 octobre 2020 ; date d'acceptation : 8 juin 2021 ; date de publication : 1er décembre 2021.
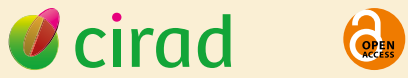

Citer l'article / To cite the article

Ouédraogo P., Traoré S., Nacoulma B. M. I., Daboue E., Bationo B. A., 2021. Dissémination et germination de semences issues des fèces de bétail au Sahel du Burkina Faso. Bois et Forêts des Tropiques, 350 : 15-27. Doi : https://doi.org/10.19182/bft2021.350.a36826 


\section{RÉSUMÉ}

\section{Dissémination et germination de semences issues des fèces de bétail au Sahel du Burkina Faso}

Les pratiques agropastorales présentent à la fois des effets positifs et négatifs sur la régénération des agroécosystèmes. Cette étude a pour objectif de déterminer l'effet des fèces de différents ruminants sur la dissémination et la germination des espèces en fonction de leurs traits fonctionnels. Ainsi, les fèces des bovins (Bos indicus), des caprins (Capra hircus) et des ovins (Ovis aries) ont été échantillonnées en 15 répétitions suivant trois périodes saisonnières de l'année dans 45 enclos en zone sahélienne du Burkina Faso. Le potentiel en semences et la capacité de germination de cinq espèces agroforestières (Balanites aegyptiaca, Faidherbia albida, Piliostigma reticulatum, Vachellia nilotica et Ziziphus mauritiana) et d'une espèce invasive (Senna obtusifolia) y ont été déterminés. Les résultats montrent que les fèces des caprins contiennent plus de semences des espèces agroforestières tandis que les ovins disséminent plus les semences de l'espèce invasive. Pour l'ensemble des six espèces, le potentiel séminal des fèces des caprins représente $64 \%$, contre $34 \%$ pour les fèces des ovins et $2 \%$ pour les fèces des bovins, de l'ensemble du potentiel séminal. Le potentiel séminal fluctue tout au long de l'année et est plus élevé en saison sèche et froide, soit $73 \%$, contre $21 \%$ pour les fèces de la saison sèche et chaude et $6 \%$ pour les fèces de la saison pluvieuse. Les semences témoins et celles issues des fèces des caprins ont les délai, durée, vitesse et taux de germination les plus élevés comparativement aux semences issues des fèces des ovins et des bovins. Les fèces assurent la dissémination et influencent les capacités germinatives des semences, d'où le rôle crucial des pratiques agropastorales dans les traits de régénération des agroécosystèmes.

Mots-clés : bovins, caprins, fèces, germination, ovins, potentiel séminal, Burkina Faso.

\section{ABSTRACT}

\section{Dissemination and germination of seeds from Sahelian cattle faeces in Burkina Faso}

Agropastoralism has both positive and negative effects on the regeneration of agro-ecosystems. This study aims to determine the effects of the faeces of different ruminants on the dissemination and germination of species according to their functional traits. The faeces of cattle (Bos indicus), goats (Capra hircus) and sheep (Ovis aries) were sampled in 15 replicates at three seasonal periods of the year in 45 enclosures in the Sahelian zone of Burkina Faso. The seed potential and germination capacity of five agroforestry species (Balanites aegyptiaca, Faidherbia albida, Piliostigma reticulatum, Vachellia nilotica and Ziziphus mauritiana) and one invasive species (Senna obtusifolia) were determined. The results show that goat faeces contain more seeds of the agroforestry species while sheep disseminate more seeds of the invasive species. For all six species, the seed bank potential in goat faeces is $64 \%$ of the total potential as against $34 \%$ in sheep faeces and $2 \%$ in cattle faeces. This potential fluctuates throughout the year and is highest in faeces produced in the cool dry season, at $73 \%$ as against $21 \%$ in the warm dry season and $6 \%$ in the wet season. The control seeds and those from goat faeces had a longer germination time and duration and a greater speed and rate of germination than those in sheep and cattle faeces. Faeces ensure dissemination and influence the germination capacity of seeds, hence the crucial role of agropastoral practices in the regeneration characteristics of agro-ecosystems.

Keywords: cattle, goats, faeces, germination, sheep, seed bank potential, Burkina Faso.

\section{RESUMEN}

\author{
Diseminación y germinación de semillas \\ provenientes de heces de ganado \\ en el Sahel de Burkina Faso
}

Las prácticas agropastorales tienen efectos tanto positivos como negativos en la regeneración de los agroecosistemas. El objetivo de este estudio es determinar el efecto de las heces de diferentes rumiantes en la diseminación y germinación de las especies según sus rasgos funcionales. Así, se tomaron muestras de heces de bovinos (Bos indicus), caprinos (Capra hircus) y ovinos (Ovis aries) en 15 réplicas durante tres períodos estacionales del año en 45 recintos de la zona del Sahel de Burkina Faso. Se determinó el potencial en semillas y la capacidad de germinación de cinco especies agroforestales (Balanites aegyptiaca, Faidherbia albida, Piliostigma reticulatum, Vachellia nilotica y Ziziphus mauritiana) y una especie invasora (Senna obtusifolia). Los resultados muestran que las heces de cabruno contienen más semillas de especies agroforestales, mientras que los ovinos esparcen más semillas de la especie invasora. Para el conjunto de las seis especies, el potencial seminal de las heces de cabruno representa el $64 \%$ del potencial seminal total, frente al $34 \%$ de las heces de ovino y al $2 \%$ de las heces de bovino. El potencial seminal fluctúa a lo largo del año y es mayor en la estación seca y fría, con un $73 \%$; frente al $21 \%$ de las heces de la estación seca y cálida, y al $6 \%$ de las heces de la estación lluviosa. Las semillas de control y las provenientes de heces de cabruno son las que tienen mayor plazo, duración, velocidad y altas tasas de germinación en comparación con las semillas de heces de ovino y de bovino. Las heces aseguran la diseminación e influyen en la capacidad de germinación de las semillas, de ahí el papel crucial de las prácticas agropastorales en la regeneración de los agroecosistemas.

Palabras clave: ganado vacuno, ganado cabruno, heces, germinación, ganado ovino, potencial seminal, Burkina Faso. 


\section{Introduction}

Les agroécosystèmes sahéliens sont marqués par des associations d'arbres, d'arbustes et de cultures (Yélémou et al., 2013). Les arbres et arbustes dans ces agro-écosystèmes composites enrichissent les services d'approvisionnement, de régulation et de soutien (Belem et al., 2007 ; Traoré et al., 2011). Cependant, divers facteurs climatiques et anthropiques entraînent une dégradation accrue des écosystèmes avec des risques sur les services de base. Dans les zones sahéliennes et soudano-sahéliennes, la dégradation des terres est caractérisée à la fois par une perte de la productivité des sols (Bayen et al., 2012) et de la biodiversité (Zwarg et al., 2012). Cette dégradation est accentuée par un manque de régénération du couvert végétal accélérant le processus de l'érosion (Ouédraogo et al., 2017). Dans les zones sahéliennes, la plupart des agroécosystèmes dégradés sont dans le stade ultime, marqués par l'apparition de sols dénudés et encroûtés (Zougmoré et al., 2003). Dans ces conditions environnementales caractérisées également par des épisodes climatiques extrêmes, les populations ont adopté des pratiques d'adaptation pour restaurer et conserver les services des agroécosystèmes. Les pratiques de restauration des terres dans les agrosystèmes sont fréquemment associées à l'utilisation de la fumure organique pour améliorer la fertilité des sols, d'où le rôle primordial des pratiques agropastorales. En plus, ces différentes pratiques d'adaptation semblent être à l'origine du retour de la végétation dans les agroécosystèmes dégradés. Cependant, les pratiques agropastorales présentent à la fois des effets positifs et négatifs sur la régénération des écosystèmes car les herbivores détruisent les plantules (Niang-Diop et al., 2011) tout en facilitant la dissémination et la levée de dormance des graines (Danthu et al., 1996 ; Guèye et al., 1999).

De nombreux fruits sont appétés par les herbivores, faisant d'eux les acteurs majeurs de la dispersion des semences par endozoochorie. Cependant, peu d'études se sont intéressées à l'effet du potentiel séminal des fèces des animaux dans la régénération des agroécosystèmes. Cette étude se base sur l'hypothèse que les matières fécales des animaux contiennent des semences des espèces végétales appétées et contribue à la régénération le long des parcours ou lors des amendements des sols utilisant la fumure organique. En plus du potentiel séminal, les fèces en tant que substrat avec des propriétés chimiques et biologiques particulières $(\mathrm{pH}, \mathrm{C}, \mathrm{N}$, enzymes extracellulaires) peuvent influer sur la capacité germinative des semences. Les traits fonctionnels des semences, notamment la dormance et la dissémination, influencent les traits de régénération (Cornelissen et al.,
2003). Effectivement, la régénération peut être limitée par des facteurs comme la dissémination, la dormance, la prédation ou l'infestation des graines (Niang-Diop et al., 2011). La plupart des graines dans la zone sahélo-soudanienne ont des téguments durs imperméables à l'eau, ce qui est le facteur le plus important pour déclencher le processus de germination. Ces semences, dites à dormance tégumentaire, sont caractéristiques des légumineuses (Fabaceae-Cesalpinoidae, Fabaceae-Mimosoidae et Fababceae-Faboidae) qui dominent la flore de notre zone d'étude (Ouédraogo et al., 2017). Parmi ces légumineuses à graines dures figurent des espèces comme Piliostigma reticulatum (DC.) Hochst., Vachellia nilotica (L.) P.J.H. Hurter \& Mabb., Faidherbia albida (Delile) A. Chev. et Senna obtusifolia (L.) Irwin \& Barneby. D'autres comme Balanites aegyptiaca (L.) Delile (Abdou Habou et al., 2019) et Ziziphus mauritiana (Lam.) (Guèye et al., 1999) sont des drupes à parois lignifiées imperméables à l'eau. La dormance tégumentaire des graines pourrait cependant être levée par le transit intestinal des ruminants dû à la mastication dentaire et aux sucs digestifs.

Notre étude portera sur ces sept espèces qui sont les principales espèces fourragères dont les fruits sont appétés par le bétail (Ouédraogo et al., 2017). Les espèces comme $P$. reticulatum (Yélémou et al., 2013) et $Z$. mauritiana à port arbustif jouent un rôle important dans la lutte contre l'érosion éolienne surtout en saison sèche. $B$. aegyptiaca et $V$. nilotica sont les principales espèces des zones de pâturage épargnées dans les champs pour servir d'ombrage et pour d'autres usages multiples. F. albida est une espèce à haute valeur fourragère (Ouédraogo et Alexandre, 1996) et agroforestière améliorant la fertilité des sols et les rendements des cultures (Depommier, 1992). S. obtusifolia, bien qu'elle soit une espèce fourragère et alimentaire, semble être invasive (Gebreyesus, 2017).

La présente étude a pour objectif de déterminer l'effet des pratiques agropastorales, notamment l'effet des matières fécales des ruminants d'élevage sur la régénération de la végétation des agroécosystèmes. Plus spécifiquement, elle vise à déterminer le potentiel en semences de $B$. aegyptiaca, Faidherbia albida, P. reticulatum, V. nilotica, Z. mauritiana et $S$. obtusifolia dans les différents types de fèces des ruminants (caprins, ovins et bovins), à tester la capacité de germination des semences issues des types de fèces, enfin à déterminer l'influence des traits fonctionnels des semences sur la régénération et les changements de la diversité des espèces dans les agroécosystèmes sahéliens. 


\section{Matériel et méthodes}

\section{Localisation et caractéristiques agroécologiques des sites d'étude}

L'étude a été effectuée dans trois villages (Sillia, Ramdolla, Tibtenga) situés en zone sahélienne du Burkina Faso appartenant à la grande bande agroécologique du Sahel (figure 1). Le climat est du type sahélien caractérisé par une saison sèche de 9 mois qui va d'octobre à juin et une saison pluvieuse de 3 mois allant de juillet à septembre. La pluviométrie annuelle fluctue entre 600 et 700 $\mathrm{mm}$. Les précipitations moyennes annuelles au cours des 20 dernières années (2000 à 2019) sont de 702 mm. Les températures les plus basses sont observées en décembre et janvier avec une moyenne de $26^{\circ} \mathrm{C}$ tandis que les plus élevées sont enregistrées entre mars et mai avec une moyenne de $43^{\circ} \mathrm{C}$. Les modes d'occupation des terres sont largement représentés par des agroécosystèmes composites constitués de champs et terres dégradées (photo 1) en extension au détriment des fourrés et des brousses tigrées.

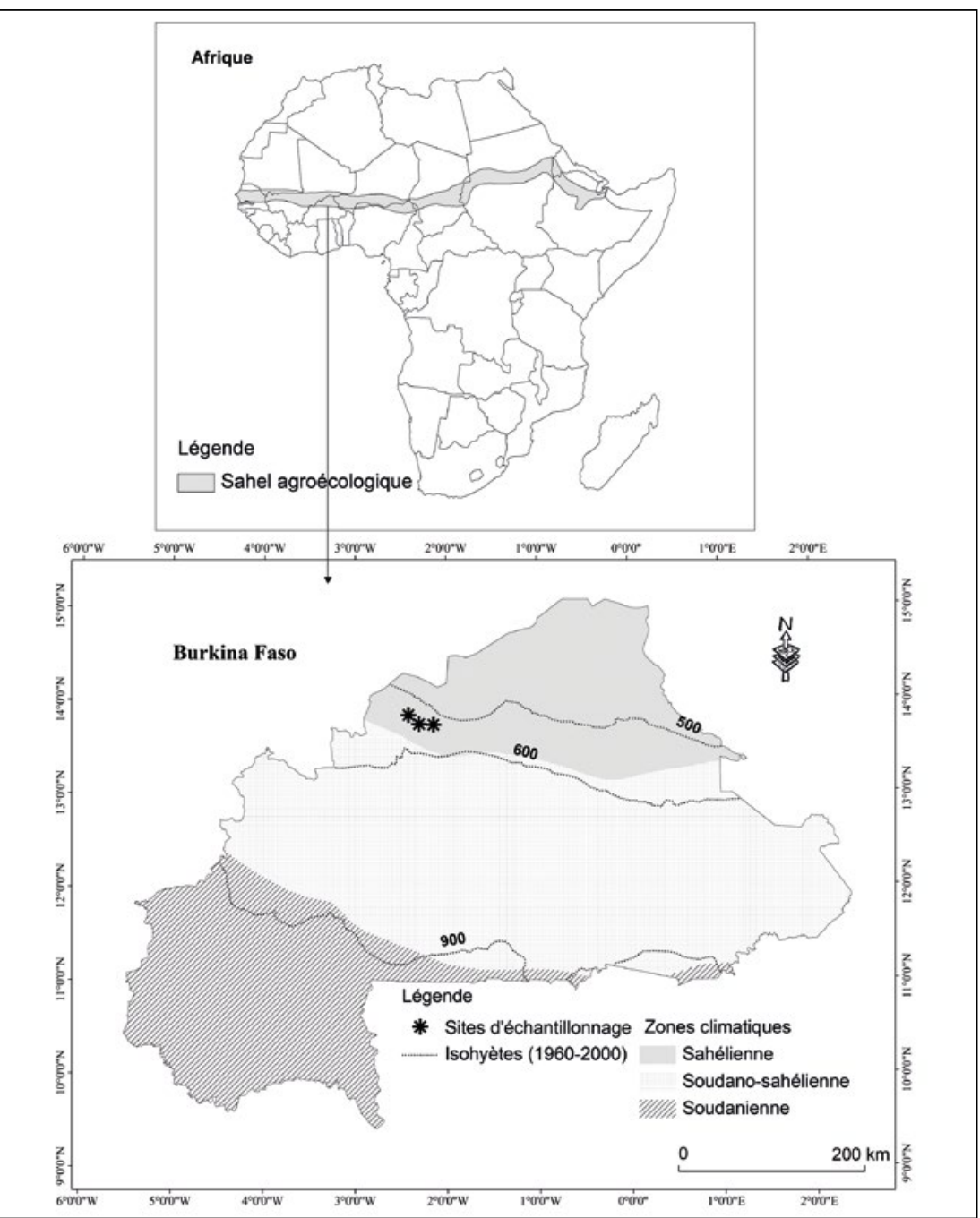

Figure 1.

Carte de localisation des sites de collecte des matières fécales. Location map of faecal collection sites.
Les principales espèces agroforestières sont disséminées à faible densité dans les champs (Adansonia digitata (L.), V. nilotica, B. aegyptiaca, Faidherbia albida, Lannea microcarpa Engl. \& K. Krause, Sclerocarya birrea (A. Rich.) Hochst., Tamarindus indica (L.), Vitellaria paradoxa C.F. Gaertn.). L'élevage, de type extensif et dépendant des ressources naturelles (pâturages, points d'eau), concerne les bovins, les petits ruminants comme les ovins et les caprins ainsi que la volaille (poules, pintades). En saison sèche, en plus des ressources naturelles, les ovins sont nourris par les fanes d'arachide, de niébé et de pois de terre, le son des céréales et par des feuilles étêtées de Pterocarpus lucens à l'approche de la saison des pluies. Les bovins sont nourris avec les tiges de mil et de sorgho en saison sèche et les feuilles d'Adansonia digitata en début de saison pluvieuse, tandis que les caprins ne reçoivent presque pas de supplément alimentaire. Par manque de fertilisants minéraux et de disponibilité en ressources fourragères, les populations pratiquent traditionnellement l'association agriculture-élevage (pratiques agropastorales).

\section{Échantillonnage et collecte des fèces}

Trois types de fèces issues des ruminants d'élevage ont été échantillonnés. Il s'agit des fèces des caprins (Capra hircus), des ovins (Ovis aries) et des bovins (Bos indicus). Chaque type de fèces ( $5 \mathrm{~kg}$ en masse sèche) a été échantillonné dans 15 enclos à raison de cinq enclos distincts dans chacune des trois localités. Chaque enclos renferme des individus de la même espèce animale (photo 2). Les fèces ont été collectées en trois périodes pour tenir compte des pratiques de stockage et de la phénologie des espèces végétales. En effet, les paysans commencent le stockage des matières fécales en saison pluvieuse, en juillet de l'année en cours jusqu'à juin de l'année suivante. Les trois périodes d'échantillonnage sont octobre (fin de saison pluvieuse), janvier (milieu de saison sèche) et juin (début de saison pluvieuse). Une fois au laboratoire, les échantillons de fèces collectées ont fait l'objet de tamisage pour collecter les semences.

\section{Détermination des caractéristiques chimiques des matières fécales}

Les échantillons de fèces ont été analysés au laboratoire pour déterminer le $\mathrm{pH}$, le carbone et l'azote. Le pHeau a été mesuré dans une suspension d'eau dans un rapport sol/solution de 1/2,5. La teneur en carbone total (C) a été déterminée par combustion sèche automatisée en utilisant un analyseur d'éléments CHN (Carlo Erba EA 1108). Le tableau I présente les caractéristiques des différentes fèces. 


\section{Détermination du potentiel séminal par type de fèces}

Chaque échantillon de matière fécale (5 kg de masse sèche) est d'abord soumis à un premier tamisage permettant de trier les grosses semences (B. aegyptiaca, Sclerocarya birrea, Z. mauritiana). Ensuite, la fraction grossière issue du premier tamisage est soumise à des tamisages successifs. Le tamisage a été effectué à l'aide d'un tamis de $2 \mathrm{~mm}$ de maille. Les semences sont ensuite triées, identifiées visuellement puis comptées par espèce

\section{Test de germination}

\section{Sélection des semences}

Les espèces ont été sélectionnées en fonction de l'importance de leur potentiel séminal. Ce sont des espèces agroforestières ( $P$. reticulatum, $Z$. mauritiana, V. nilotica, F. albida et $B$. aegyptiaca) et invasive (S. obtusifolia) (tableau II).

La capacité germinative des semences de ces espèces issues des fèces de la saison sèche et froide (période à potentiel séminal maximal) a été testée avec leurs témoins. Les semences témoins ont été échantillonnées en récoltant les fruits des espèces sélectionnées sur leurs semenciers. Pour obtenir les semences, les gousses de $P$. reticulatum, $V$. nilotica et $F$. albida ont été extraites suivant le protocole d'extraction utilisé par le Centre national de semences forestières (CNSF). Les gousses de $S$. obtusifolia étant déhiscentes, les semences ont été libérées par l'ouverture de leurs fentes. Les semences obtenues ont fait l'objet d'un tri pour éliminer les

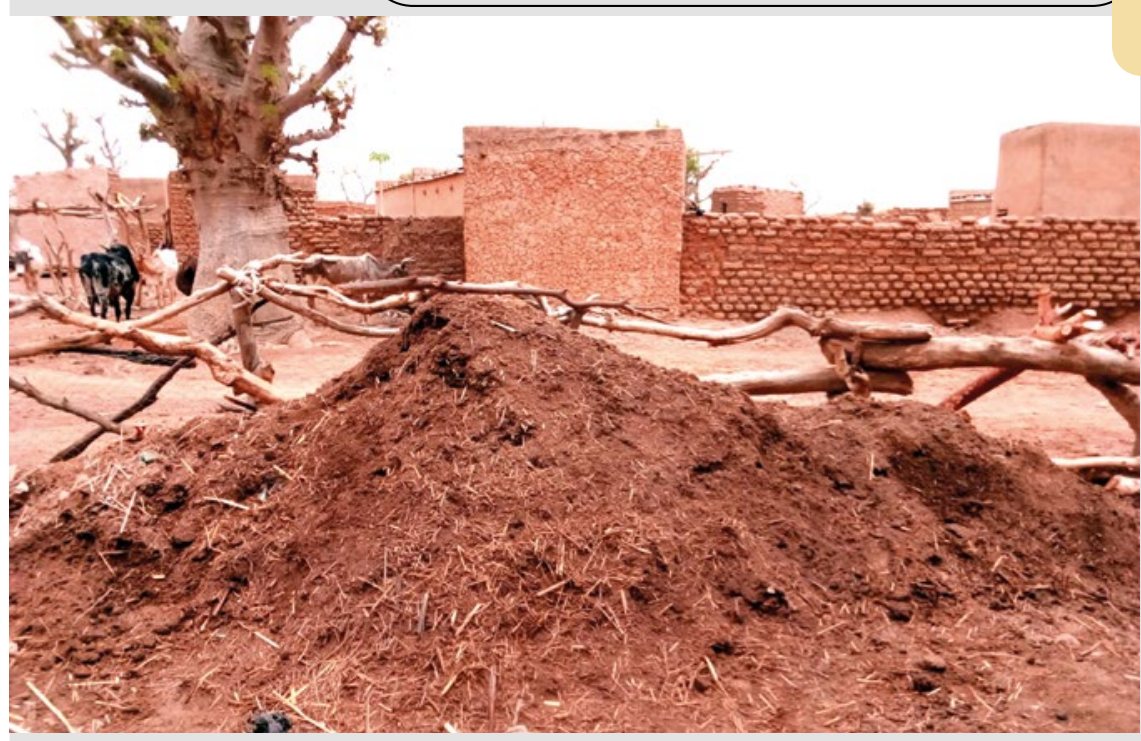

Photo 2.

Matières fécales de bovins dans une étable.

Cattle faeces in a cowshed.

Photo P. Ouédraogo. 
semences de mauvaise qualité. Pour chaque espèce, 12 lots des semences issues des types de fèces (caprins, ovins et bovins) et des semenciers des trois localités (Ramdolla, Sillia et Tibtenga) ont été constitués pour effectuer les tests de germination. Les semences de chaque lot de semences ont été soigneusement triées en éliminant les semences brisées, infectées par les insectes, pourries et immatures. Ensuite, les semences ont été homogénéisées, puis le choix des semences à tester dans le lot s'est fait suivant la méthode des divisions progressives (Niang-Diop et al., 2011). Le nombre de semences par lot varie de 15 à 20 selon les espèces. Pour un lot donné, si le nombre de semences qualitatives est inférieur au nombre voulu (15 ou 20), les semences de ce lot ne sont pas prises en compte.

\section{Dispositif expérimental et suivi de la germination}

Les semis ont été effectués dans des bacs de germination contenant $1 / 5$ de sable, $3 / 5$ de terre et $1 / 5$ de compost bovin à raison de 10 semences par bac pour $B$. aegyptiaca, $P$. reticulatum, $S$. obtusifolia et $Z$. mauritiana et 7 à 8 semences pour $V$. nilotica et Faidherbia albida. Les semences ont été semées à une profondeur de 2 à $3 \mathrm{~cm}$ environ et espacées entre elles d'environ $3 \mathrm{~cm}$. L'arrosage a été effectué chaque matin et les germinations ont été enregistrées quotidiennement. Nous avons considéré qu'une semence a germé lorsque nous constations l'émergence de la tigelle (levée). La durée de suivi est de 4 mois. Les paramètres de germination suivants ont été déterminés :

- le temps de latence (temps mis entre le semis et l'apparition de la première radicule) ;

- le temps moyen de germination (temps à la fin duquel on atteint $50 \%$ du taux de germination totale du lot de semences testées) ;

- la durée de germination (temps entre la première et la dernière germination) ;

- le taux de germination ou capacité germinative ou encore pouvoir germinatif (proportion de semences ayant germé pendant la durée de l'observation), déterminé par la formule suivante :

Taux de germination $=\frac{\text { Nombre de graines germées }}{\text { Nombre total de graines semées }} \times 100$

- le taux maximum de germination journalière (TMGj), obtenu par le calcul du pourcentage de germination maximale obtenu en un jour au cours de la durée de l'observation ;

- le délai de germination maximale obtenu par le comptage du nombre de jours après semis où l'on a obtenu le maximum de germination journalière.

\section{Analyse statistique}

Les données sur les variables quantitatives ont fait l'objet d'analyses statistiques descriptives (moyennes, écarts-types, erreur standard) et inférentielles en utilisant les modèles linéaires généralisés (GLM). Ces modèles permettent d'ajuster les variables avec les types de distributions usuels. Ainsi les données de comptage (potentiel séminal) ont-elles été ajustées à la distribution de quasi-Poisson (dispersion élevée), les données de proportion (taux de germination) à la distribution binomiale et celles sur la durée (délai, vitesse, durée, délai maximum de germination) et le taux de germination maximal à la distribution gamma. Le meilleur ajustement des données est retenu suite à l'examen des graphiques diagnostiques. En présence d'effets significatifs de l'origine des semences ( $p \leq 0,05)$, une comparaison post-hoc ( 2 à 2 ) est effectuée ensuite en utilisant le test de Tukey à un niveau de signification de $5 \%$. Une analyse en composantes principales (ACP) a également été réalisée afin de catégoriser les paramètres de germination qui caractérisent les espèces végétales et les types de semences. Les analyses ont été faites en utilisant le logiciel $\mathrm{R}$, version 3.6.0.

\section{Résultats}

\section{Potentiel séminal dans les fèces}

Pour l'ensemble des six espèces, le potentiel séminal des fèces des caprins représente $64 \%$, contre $34 \%$ pour les fèces des ovins et $2 \%$ pour les fèces des bovins, de l'ensemble du potentiel séminal. Suivant les périodes de collecte, le potentiel séminal dans les fèces collectées en saison sèche et froide représente $73 \%$ contre $21 \%$ en saison sèche et chaude et $6 \%$ durant la saison pluvieuse. Pour l'ensemble des matières fécales et sur un cumul des trois périodes de collecte, les semences de $P$. reticulatum représentent $38 \%$ du potentiel séminal, celles de Z. mauritiana $20 \%$, celles de V. nilotica $17 \%$, celles de S. obtusifolia $14 \%$, celles de F. albida $6 \%$ et celles de B. aegyptiaca $4 \%$. Le potentiel séminal de la saison sèche et froide est significativement plus élevé que celui de la saison pluvieuse et de la saison sèche et chaude $(P<0,05)$. En saison pluvieuse, aucune semence n'a été trouvée dans les fèces des bovins (tableau III). II n'existe pas de différence significative dans le potentiel séminal des espèces agroforestières contenues dans les fèces des caprins et dans celles des ovins $(P>0,05)$ à cette période. Cependant, les semences de $S$. obtusifolia (espèce invasive) sont beaucoup plus abondantes dans les fèces des ovins, soit plus de six fois les fèces des caprins. En saison sèche et froide, les fèces des caprins, des ovins et des bovins ont montré des effets significativement différents dans la dissémination des semences des espèces étudiées $(P<0,05)$. À cette période, les semences de $P$. reticulatum représentent $38 \%$ du potentiel séminal, celles de Z. mauritiana $24 \%$, celles de V. nilotica $21 \%$, celles de S. obtusifolia $9 \%$, celles de F. albida $5 \%$ et celles de $B$. aegyptiaca $3 \%$. De même, les fèces des caprins contiennent respectivement $29,45,154,65$ et 65 fois plus les semences de $P$. reticulatum, $Z$. mauritiana, $V$. nilotica, F. albida et $B$. aegyptiaca que les fèces des bovins et respectivement 2, 11, 8 et 8 fois plus que celles des ovins (tableau III). Les fèces des ovins contiennent à cette période 12 fois plus les semences de $S$. obtusifolia que les fèces des caprins et 8 fois celles des bovins. En saison sèche et chaude, les fèces des caprins contiennent davantage les semences des espèces agroforestières $(\mathrm{P}<0,05)$ à l'exception de $B$. aegyptiaca et $P$. reticulatum tandis que les ovins disséminent plus les semences de $S$. obtusifolia et $P$. reticulatum. Pendant cette période, les semences de $P$. reticulatum représentent 
Tableau III.

Potentiel séminal moyen (nombre de semences $/ \mathrm{kg}$ ) en fonction de la source des matières fécales et de la période de collecte.

Average seminal potential (number of seeds/ $\mathrm{kg}$ ) depending on the source of the faeces and the collection period.

Période
Fumier

\begin{tabular}{|c|c|c|c|}
\hline Balanites aegyptiaca & $6,10 a$ & $4,10 a$ & $\mathrm{Ob}$ \\
\hline Faidherbia albida & $0,13 a$ & $0,07 a$ & $\mathrm{Ob}$ \\
\hline Piliostigma reticulatum & $1,20 \mathrm{a}$ & $0,27 a$ & $\mathrm{Ob}$ \\
\hline Senna obtusifolia & $4,28 b$ & $28,47 a$ & Oc \\
\hline Vachellia nilotica & $0,73 a$ & $0,13 a$ & $\mathrm{Ob}$ \\
\hline Ziziphus mauritiana & $0,47 a$ & $0,28 a$ & $\mathrm{Ob}$ \\
\hline
\end{tabular}

Saison pluvieuse

Caprins Ovins Bovins

$0,28 a \quad 0 b$
Saison sèche et chaude

\begin{tabular}{|c|c|c|c|c|c|}
\hline \multicolumn{3}{|c|}{ Saison sèche et froide } & \multicolumn{3}{|c|}{ Saison sèche et chaude } \\
\hline Caprins & Ovins & Bovins & Caprins & Ovins & Bovin \\
\hline $17,57 a$ & $2,11 b$ & $0,27 c$ & $4,38 a b$ & $2,05 b$ & $0,33 b$ \\
\hline $26,88 a$ & $3,15 b$ & $0,41 c$ & $21,34 a$ & $2,27 b$ & $0,37 b$ \\
\hline $156,36 a$ & $64,60 \mathrm{~b}$ & $5,4 \mathrm{C}$ & $49,11 b$ & $55,38 a$ & $2,17 c$ \\
\hline $3,69 b$ & $45,17 a$ & $5,32 b$ & $2,03 b$ & $31,62 a$ & $1,59 \mathrm{~b}$ \\
\hline $112,64 a$ & $10,24 b$ & $0,73 c$ & $18,86 a$ & $1,64 b$ & $0,40 b$ \\
\hline $93,08 a$ & $46,27 b$ & $2,07 c$ & $17,68 a$ & $5,38 b$ & $0,35 c$ \\
\hline
\end{tabular}

Pour chaque espèce et par période, les moyennes suivies de la même lettre ne sont pas significativement différentes $(P>0,05)$. Les comparaisons sont faites entre les différentes espèces animales pour chaque espèce végétale par période.

Tableau IV.

Paramètres de germination en fonction des espèces.

Germination parameters according to species.

\begin{tabular}{|c|c|c|c|c|c|c|c|}
\hline Espèce & Fumier & $\begin{array}{l}\text { Dél.ger } \\
\text { (jours) }\end{array}$ & $\begin{array}{c}\text { Tem.moy.ger } \\
\text { (jours) }\end{array}$ & $\begin{array}{l}\text { Dur.ger } \\
\text { (jours) }\end{array}$ & $\begin{array}{c}\text { Tmax.ger.jour } \\
\text { (\%) }\end{array}$ & $\begin{array}{l}\text { Dél.max.ger } \\
\text { (jours) }\end{array}$ & $\begin{array}{c}\text { T.ger.général } \\
(\%)\end{array}$ \\
\hline \multirow{3}{*}{ Balanites aegyptiaca } & Caprin & $13 a$ & $16 a$ & $17 \mathrm{~b}$ & $11,67 b$ & $16 \mathrm{~b}$ & $50 a$ \\
\hline & Ovin & $13 a$ & $17 a$ & $7 c$ & $10 c$ & $15 b$ & $47,5 a$ \\
\hline & Témoin & $13 a$ & $17 a$ & $29 a$ & $16,67 a$ & $17 a$ & $46,67 a$ \\
\hline \multirow{4}{*}{ Faidherbia albida } & Caprin & $7 c$ & $31 a$ & $102 a$ & $6,67 c$ & $25 b$ & $88,89 b$ \\
\hline & Ovin & $13 b$ & $25 c$ & $87 c$ & $20 a$ & $25 b$ & $93,33 a$ \\
\hline & Bovin & $20 a$ & $27 \mathrm{~b}$ & $42 d$ & $20 a$ & $26 b$ & $86,67 c$ \\
\hline & Témoin & $8 c$ & $32 a$ & $95 b$ & $8,89 b$ & $34 a$ & $82,22 d$ \\
\hline \multirow{4}{*}{ Piliostigma reticulatum } & Caprin & $12 b$ & $61 \mathrm{c}$ & $121 a$ & $3,33 b$ & $53 ; 60 ; 77 ; 91 d$ & $53 c$ \\
\hline & Ovin & $10 c$ & $70 a$ & $121 a$ & $6,67 a$ & $72 a$ & $60 b$ \\
\hline & Bovins & $10 c$ & $70 a$ & $108 c$ & $7,5 a$ & $69 \mathrm{~b}$ & $53 c$ \\
\hline & Témoin & $14 a$ & $65 b$ & $118 \mathrm{~b}$ & $6,67 a$ & $64 ; 66 c$ & $68 a$ \\
\hline \multirow{4}{*}{ Vachellia nilotica } & Caprin & $19 a$ & $23 c$ & $11 \mathrm{c}$ & $4,44 \mathrm{C}$ & $23 a$ & $11,11 \mathrm{c}$ \\
\hline & Ovin & $10 d$ & $34 a$ & $25 b$ & $3,33 c$ & $10 c$ & $10 c$ \\
\hline & Bovin & $12 \mathrm{c}$ & $18 d$ & $12 c$ & $10 a$ & $18 \mathrm{~b}$ & $20 b$ \\
\hline & Témoin & $15 b$ & $25 a$ & $83 a$ & $6,7 b$ & $18 \mathrm{~b}$ & $35,56 a$ \\
\hline \multirow{4}{*}{ Ziziphus mauritiana } & Caprin & $11 \mathrm{c}$ & $17 a$ & $47 a$ & $11,67 a$ & $16 \mathrm{~b}$ & $63,33 a$ \\
\hline & Ovin & $13 b$ & $17 a$ & $35 b$ & $12,5 a$ & $13 c$ & $55 b$ \\
\hline & Bovin & $12 \mathrm{cb}$ & $17 a$ & $19 c$ & $10 \mathrm{~b}$ & $16 \mathrm{~b}$ & $62,5 a$ \\
\hline & Témoin & $15 a$ & $18 a$ & $36 b$ & $10 \mathrm{~b}$ & $18 a$ & $45 c$ \\
\hline \multirow{4}{*}{ Senna obtusifolia } & Caprin & $25 a$ & $61 \mathrm{c}$ & $74 d$ & $1,67 c$ & $25 a$ & $16,67 c$ \\
\hline & Ovin & $9 c$ & $42 d$ & $90 b$ & $3,33 b$ & $9 c$ & $28,33 b$ \\
\hline & Bovin & $8 c$ & $76 a$ & $93 a$ & $1,67 c$ & $8 c$ & $13,33 d$ \\
\hline & Témoin & $12 b$ & $69 \mathrm{~b}$ & $90 \mathrm{~b}$ & $5 a$ & $12 b$ & $38,33 a$ \\
\hline
\end{tabular}

Dél.ger = délai de germination ; Vit.ger = vitesse de germination ; Dur.ger = durée de germination ; Dél.ger.max = délai de germination maximal; Tmax.ger.jour = taux maximum de germination journalière ; T.ger.général = taux général de germination. 


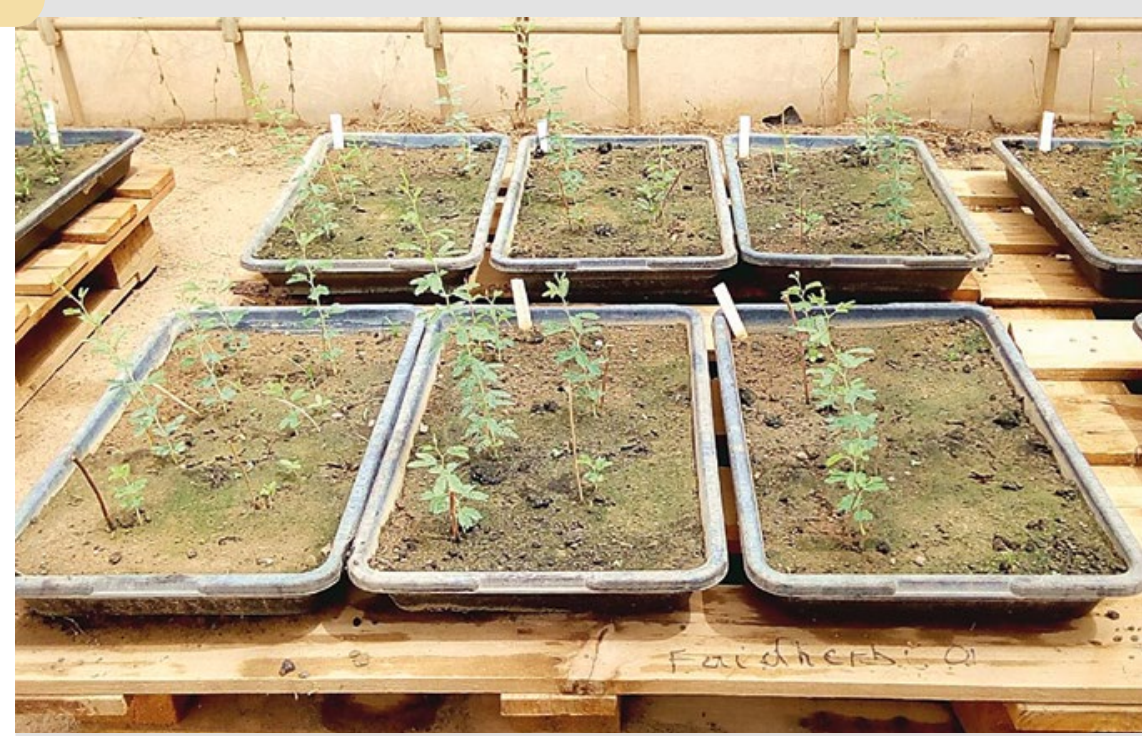

Photo 3.

Plantules de Faidherbia albida.

Faidherbia albida seedlings.

Photo P. Ouédraogo.

$49 \%$ du potentiel séminal contre $15 \%$ pour S. obtusifolia, $12 \%$ pour Faidherbia albida, $11 \%$ pour Z. mauritiana, $10 \%$ pour $V$. nilotica et $3 \%$ pour $B$. aegyptiaca. Les fèces des ovins contiennent respectivement 20 et 25 fois plus les semences de $S$. obtusifolia et de $P$. reticulatum que les fèces des bovins et respectivement 16 et une fois plus que celles des caprins. Les fèces des caprins contiennent respectivement 58, 50, 47 et 13 fois plus les semences de Faidherbia albida, Z. mauritiana, $V$. nilotica et $B$. aegyptiaca que les fèces des bovins et $9,3,11$ et 2 fois plus que celles des ovins.

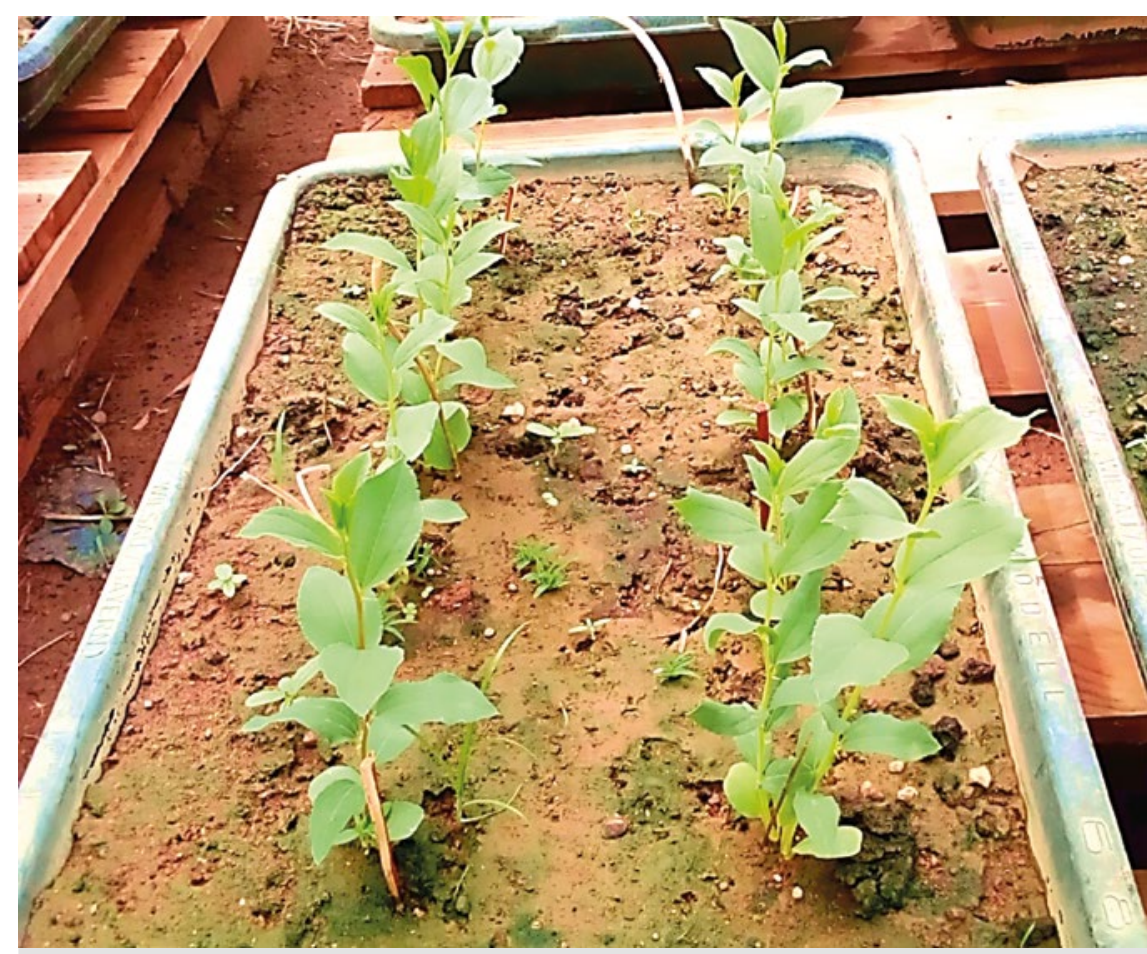

Photo 4.

Plantules de Ziziphus mauritiana.

Ziziphus mauritiana seedlings.

Photo P. Ouédraogo.

\section{Paramètres de germination en fonction des semences par type de matière fécale}

Les photos 3 et 4 montrent des plantules de $F$. albida et $Z$. mauritiana après germination des graines. Il n'y a pas de différence significative entre les taux de germination des semences de $B$. aegyptiaca issues des fèces et celui des semences témoins (tableau IV). Le transit intestinal des bovins, des caprins et des ovins a permis d'avoir des taux de germination des semences de F. albida plus élevés de 4,7 et $11 \%$ comparativement aux semences témoins. Chez Z. mauritiana, les semences issues des fèces des ovins, des bovins et des caprins ont des taux de germination plus élevés respectivement de 10,18 et $18 \%$ comparativement aux semences témoins. Le taux de germination des semences témoins de $P$. reticulatum est plus élevé de 8,15 et $15 \%$ que celui des semences issues respectivement des fèces des ovins, des bovins et des caprins. Aussi, le taux de germination des semences témoins de $V$. nilotica est plus élevé de 16, 24 et $26 \%$ que celui des semences issues respectivement des fèces des bovins, des caprins et des ovins, tandis que le taux de celles de $S$. obtusifolia est plus élevé de 10, 22 et $25 \%$ par rapport aux semences issues respectivement des fèces des ovins, des caprins et des bovins.

L'analyse en composantes principales (ACP) permet de classer les espèces en quatre groupes (figure 2). Le groupe 1 est constitué de $B$. aegyptiaca, $F$. albida et Z. mauritiana. Le groupe 2 est représenté par l'espèce $P$. reticulatum, le groupe 3 par $S$. obtusifolia et le groupe 4 par $V$. nilotica. Les deux premiers axes concentrent plus de $73 \%$ de la variance totale. L'axe 1 représente $43,59 \%$ de l'information et le deuxième axe $29,78 \%$. Les figures $2 a$ et $2 b$ montrent que $B$. aegyptiaca, F. albida et $Z$. mauritiana sont caractérisées par les plus forts taux de germination maximale journalière comparativement aux trois autres espèces (tableau IV). Faidherbia albida se distingue aussi de ces deux autres espèces par son fort taux de germination dépassant $80 \%$ quel que soit le type de semences. $P$. reticulatum se distingue des autres espèces par la longue durée de germination (108-121 jours) et le plus long délai de germination maximale (53-91 jours) de ses semences. Senna obtusifolia et $V$. nilotica se caractérisent par leur faible taux de germination. En analysant les courbes de germination, après deux semaines de temps de latence, les semences de $B$. aegyptiaca (figure $3 a$ ) présentent une germination rapide se caractérisant par une faible durée de ger- 
mination. La courbe de germination de F. albida montre une forte germination des semences qui diminue à partir du $50^{\mathrm{e}}$ jour après semis (figure $3 \mathrm{~b}$ ). La courbe de germination de $P$. reticulatum (figure $3 c$ ) présente des escaliers dont la longueur de stabilité est courte avec de faibles hauteurs. La courbe de germination de V. nilotica (figure $3 d$ ) présente des escaliers dont la longueur de stabilité est longue avec de faibles hauteurs. La courbe de germination de $Z$. mauritiana (figure 3 e) présente la même allure que celle de B. aegyptiaca et celle de S. obtusifolia (figure $3 \mathrm{f}$ ) la même allure que la courbe de germination de $V$. nilotica.

\section{Discussion}

\section{Potentiel séminal des espèces dans les différents types de matières fécales}

Les résultats ont révélé que les fèces des caprins ont un potentiel séminal plus élevé que celles des ovins dont le potentiel est supérieur à celui des bovins. Cette différence dans la dissémination des semences pourrait s'expliquer par leurs préférences alimentaires (Dumont, 1996 ; Rutter, 2010), leur système buccal et digestif, la disponibilité et l'accessibilité des espèces appétées (César et Zoumana, 1999). Les études de César et Zoumana (1999) ont rapporté que les caprins se nourrissent principalement de ligneux et subligneux (80\%) ; les ovins ont le régime le plus équilibré (60\% de graminées, $20 \%$ d'autres herbes et $20 \%$ de ligneux et subligneux) et les bovins consomment principalement les graminées (90\%). Les fèces des ovins se distinguent par un potentiel séminal marqué par l'abondance des semences de S. obtusifolia qui est une espèce invasive (Gebreyesus, 2017). En dehors de la saison pluvieuse, les caprins et les ovins sont en liberté dans la nature tandis que les bovins sont conduits par un berger. Cette mise en liberté en plus de leur rapidité à courir font d'eux les principaux consommateurs des gousses de F. albida comparativement aux bovins (Depommier, 1996). Aussi, l'efficacité de la dissémination des semences végétales par les herbivores dépend du pourcentage des pertes par écrasement et abrasion mécanique lors du processus de mastication et par abrasion chimique lors du processus de digestion (Suárez et Malo, 1997) qui serait plus élevé chez les bovins que chez les petits ruminants. Le système buccal des ruminants joue un rôle dans la sélection des espèces appétées. En effet, le système buccal des caprins est bien adapté à la consommation des espèces épineuses par rapport aux ovins (Ginane et al., 2008). Les bovins sont moins aptes à la consommation des espèces épineuses du fait de leur appareil buccal ina- dapté mais digèrent mieux les fourrages très fibreux en raison du plus long temps de séjour des aliments dans le rumen (Dumont et al., 2007 ; Ginane et al., 2008). Ce qui explique l'absence de semences dans les fèces des bovins en saison pluvieuse où les herbacées sont disponibles. II apparaît que le nombre de semences disséminées diffère en fonction des périodes. Balanites aegyptiaca et $S$. obtusifolia sont les semences les mieux représentées dans les matières fécales d'octobre. Les semences de $V$. nilotica, F. albida et $Z$. mauritiana sont plus représentées au mois de janvier et diminuent au mois de juin. Ces résultats reflètent la phénologie des espèces étudiées. La phénologie des espèces est un trait influençant la disponibilité donc la capacité de dissémination des semences au cours de l'année.

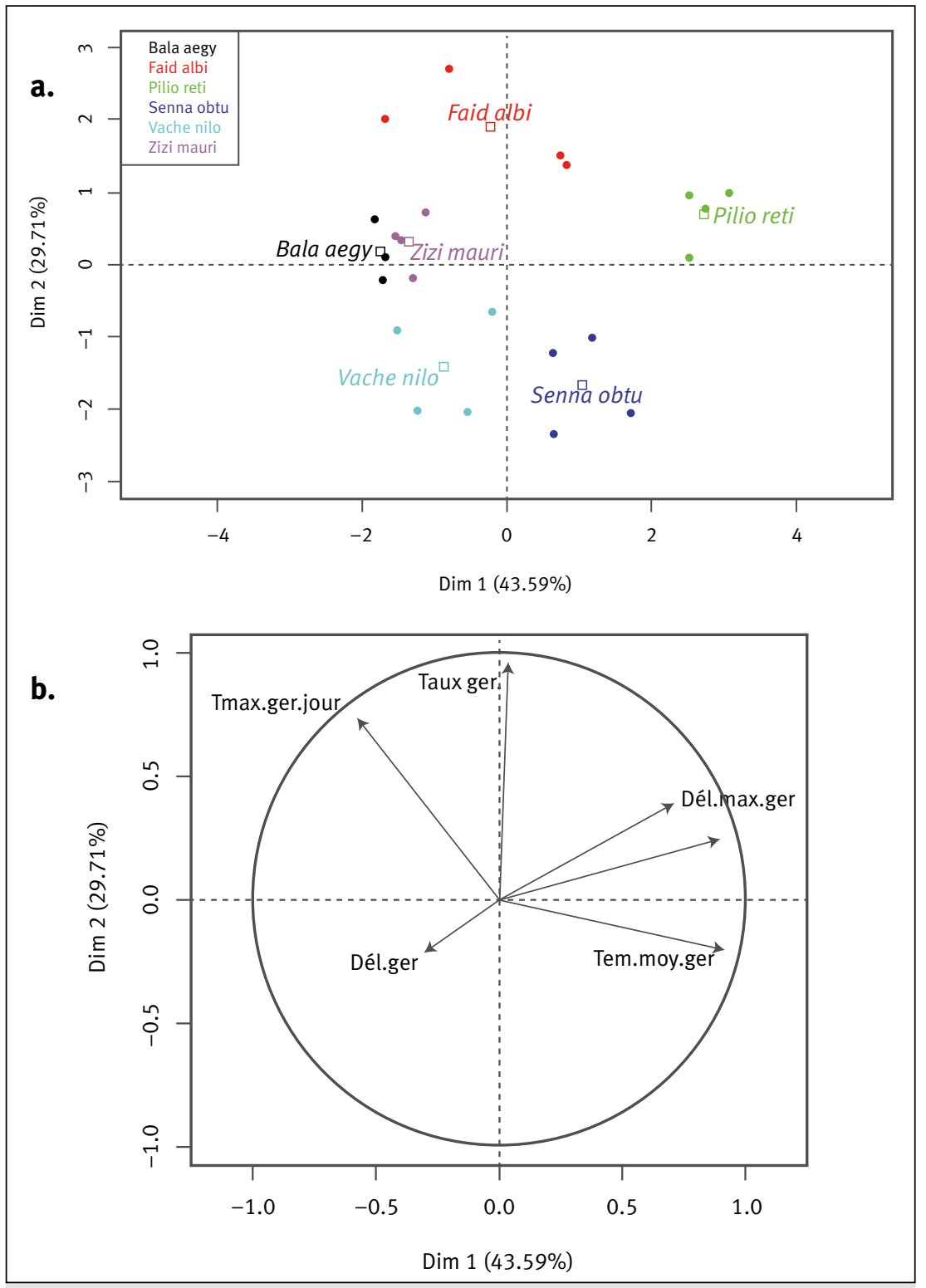

Figures 2.

(a) Plan factoriel de l'analyse en composantes principales (espèces végétales $\mathrm{x}$ traitements [types de semences]). (b) Cercle des corrélations entre paramètres de germination dans le plan principal (plan 1-2).

(a) Factorial design of the principal component analysis (plant species $x$ treatments [seed types]). (b) Circle of correlations between germination parameters in the main design (design 1-2). 

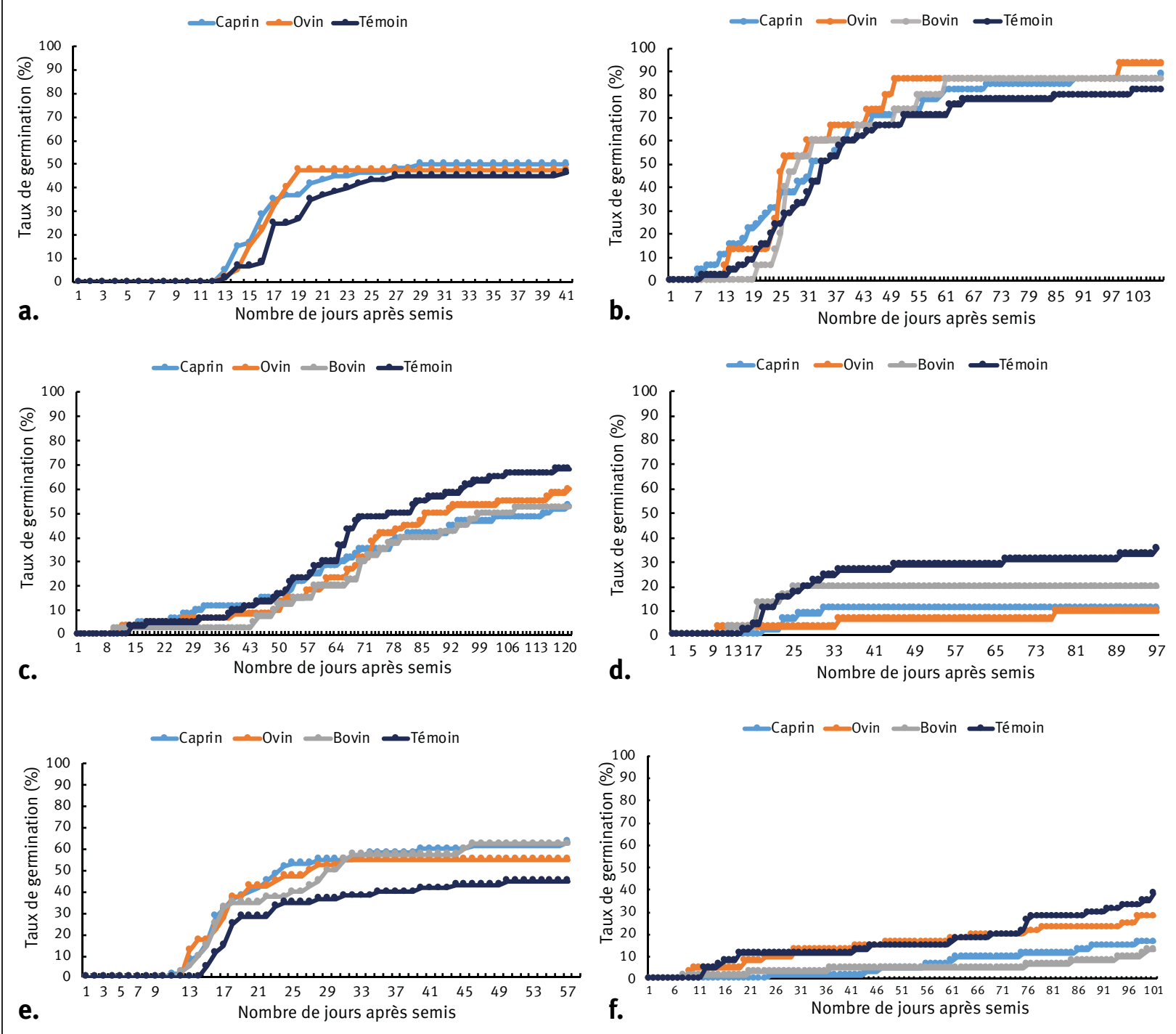

Figure 3.

Variation du taux de germination des semences issues de différents types de fèces en comparaison avec les semences témoins. (a) Balanites aegyptiaca ; (b) Faidherbia albida ; (c) Piliostigma reticulatum ; (d) Vachellia nilotica ; (e) Ziziphus mauritiana ; (f) Senna obtusifolia.

Variation in germination rate of seeds from different types of faeces in comparison with control seeds.

(a) Balanites aegyptiaca; (b) Faidherbia albida; (c) Piliostigma reticulatum; (d) Vachellia nilotica;

(e) Ziziphus mauritiana; (f) Senna obtusifolia.

\section{Influence des types de fèces sur la germination}

Le passage des semences dans le tractus digestif peut impacter, négativement ou positivement, la viabilité des semences en supprimant l'effet inhibiteur de la pulpe, en altérant chimiquement ou mécaniquement les enveloppes séminales (Samuels et Levey, 2005 ; Traveset et al., 2007 ; Bradford et Westcott, 2010). Le suc digestif, l'activité masticatoire et la durée des aliments dans le tube digestif des animaux sont des facteurs qui peuvent influer sur le devenir des semences ingérées. Considérant les paramètres (Norden et al., 2009) qui caractérisent une bonne germination (courts délai et durée de germination), ce sont les ovins et les bovins qui améliorent la germination comparativement aux caprins et aux témoins. Si les ovins améliorent la germination des semences de $V$. nilotica, $B$. aegyptiaca, $F$. albida et S. obtusifolia, cela serait lié à leur suc digestif. Les fèces des ovins ont des taux d'azote, de carbone et un rapport $\mathrm{C} / \mathrm{N}$ plus élevé que ceux des caprins avec un $\mathrm{pH}$ moins basique. Cela confirme le fait que les ovins ont un régime alimentaire intermédiaire consommant plus de fibres que les caprins (César et Zournana, 1999). Ainsi, les semences ont dû séjourner plus longtemps dans le suc digestif des ovins que les caprins. Aussi, le $\mathrm{pH}$ moins basique que celui des caprins aurait un effet positif sur la levée de la dormance comme le traitement à l'acide. Le pH et la durée de séjour ont permis donc aux sucs digestifs d'altérer chimiquement les barrières (noyaux, téguments), d'où une durée, une vitesse et un délai de germination plus réduits que chez les caprins. Aussi, le nombre élevé de semences dans les tubes digestifs des caprins n'a pas facilité leur traitement par les sucs digestifs. C'est ainsi que la germination des 
semences issues des caprins est plus échelonnée dans le temps. Les bovins consomment très peu ces semences. Les fèces des bovins ont un rapport $\mathrm{C} / \mathrm{N}>20$ et se caractérisent par un $\mathrm{pH}$ plus basique que celui des ovins et caprins. Le rapport $\mathrm{C} / \mathrm{N}$ plus élevé indique la forte consommation des fourrages fibreux par les bovins. Ce qui aurait permis aux semences de $Z$. mauritiana et $F$. albida de passer plus de temps dans le tractus intestinal des bovins car les gousses de ces deux espèces sont fibreuses, d'où une amélioration de la germination. Le faible nombre des semences dans le tube digestif des bovins aurait facilité leur traitement par les sucs digestifs. Le taux de germination élevé des semences de $V$. nilotica issues des fèces des bovins comparativement à celles issues des fèces des caprins et des ovins pourrait s'expliquer par l'intensité de la mastication car les gousses de cette espèce ne sont pas épaisses, permettant une meilleure scarification des graines comparativement aux graines de $F$. albida et $P$. reticulatum dont les gousses sont très lignifiées. En outre, les bovins ont davantage accès aux gousses présentes sur les semenciers de cette espèce que les caprins et ovins qui ont accès seulement aux gousses sèches tombées au sol. Les graines des gousses fraîches ont une plus forte teneur en eau que celles des gousses sèches qui sont très dures expliquant ainsi leur faible germination. S'agissant de Z. mauritiana, la mastication plus intense des drupes par les bovins du fait de l'endocarpe lignifié aurait permis de mieux les scarifier d'où une meilleure germination. En fait, l'effet ramollissant des sucs digestifs et l'effet de la mastication augmentent la porosité à l'eau et à l'air de l'endocarpe, permettant une germination rapide plutôt qu'un taux de germination élevé (Guèye et al., 1999 ; Abdou Habou et al., 2019).

\section{Influence des traits fonctionnels des semences sur la germination}

Les semences des espèces étudiées présentent une dormance physique. En effet, les semences de B. aegyptiaca sont des noyaux à endocarpe lignifié et fibreux renfermant une graine (amande). Celles de Z. mauritiana sont également des noyaux à endocarpe lignifié et fortement sclérenchymatisé avec une ou deux loges carpellaires contenant chacune une graine. Les semences de $V$. nilotica sont constituées d'un épiderme palissadique, d'une assise en sabliers, le reste du tégument étant formé par un tissu fibreux, très épais, dont les cellules sont arrondies, collenchymateuses, lacuno-digitées. Les semences de S. obtusifolia sont constituées d'une cuticule, de macrosclérides et ostéosclérides (Mensah et Ekeke, 2016). Les semences de F. albida sont protégées par une cuticule cireuse et celles de $P$. reticulatum présentent des enveloppes coriaces (Yélémou et al., 2007).

La dormance d'une graine est un phénomène très important qui permet aux plantes de coloniser les milieux les plus variés et de survivre à des bouleversements importants des écosystèmes. La dormance est aussi une stratégie pour résister à l'extinction d'une espèce si une perturbation exceptionnelle détruit l'ensemble des parties végétatives (Crosaz, 1995). Ainsi, elle est une stratégie écologique qui ne déclenche la germination que dans des conditions favo- rables capables d'assurer la survie des plantules. Pour ces semences, il y a nécessité de prétraitement afin d'obtenir des germinations rapides avec des taux de germination élevés et uniformes (Burrows et al., 2009 ; Azad et al., 2011).

De manière générale, il n'y a pas de différence significative entre les taux de germination des semences issues des fèces et des témoins. Selon Danthu et al. (1996), l'ingestion par les ruminants domestiques ne peut être considérée comme un facteur favorisant la levée de dormance des semences dures dont $F$. albida et $V$. nilotica mais seulement comme un moyen de dissémination des semences intactes. Concernant $B$. aegyptiaca et $Z$. mauritiana, le faible taux de germination observé chez ces deux espèces serait dû à l'endocarpe qui constitue une barrière physique à la pénétration de l'eau et de l'air dans la graine (amande) qu'il contient (Guèye et al., 1999 ; Karimpour et al., 2013 ; Elfeel et Hindi, 2014). Pour ces types de semences, l'amélioration de la germination se fait en mettant à nu les semences ou en fissurant l'endocarpe (Danthu et al., 1996 ; Guèye et al., 1999) car la levée de dormance reste limitée après mastication et passage dans les sucs digestifs des animaux (Abdou Habou et al., 2019). En mettant à nu ou en fissurant l'endocarpe, l'eau atteint l'amande qui est charnue (graine non dure) et capable de germer facilement. Par exemple, observée par les paysans, la forte présence des juvéniles de ces deux espèces dans les agrosystèmes soumis aux pratiques de conservation des eaux et des sols (zai) serait due aux meilleures conditions hydriques qu'offrent les poquets de zaï. Indépendamment de l'espèce, la nature des semences joue un rôle important dans la germination. Ce qui caractérise ces deux espèces sur le plan de la germination.

Les semences des légumineuses (Faidherbia albida, $P$. reticulatum, $S$. obtusifolia et $V$. nilotica) ont les plus longues durées de germination se caractérisant par un échelonnement dans le temps de la levée des semences, qui est d'ailleurs une stratégie d'adaptation des espèces à la forte variabilité de la pluviométrie (Bationo, 1996 ; Depommier, 1996). En fait, les semences de ces espèces nécessitent des prétraitements pour fragiliser les téguments durs et favoriser la germination (Diallo et al., 1996 ; Yélémou et al., 2007 ; Abdulazeez, 2016 ; Mensah et Ekeke, 2016 ; Satti et al., 2016). Vachellia nilotica et $S$. obtusifolia sont les espèces qui ont les plus faibles taux de germination. Faidherbia albida a un fort taux de germination quel que soit le type de semences tandis que $P$. reticulatum a un taux de germination moyen. Les semences des légumineuses, bien que dures, se distinguent par l'épaisseur de la cuticule qui recouvre le tissu palissadique (Niang-Diop et al., 2011) ayant un effet inhibiteur qui rend les semences imperméables à l'eau (Kheloufi et Mansouri, 2017). Ainsi, en fonction de l'épaisseur des téguments, il faut une période plus ou moins longue pour que la graine s'oxygène et s'hydrate pour permettre sa germination, ce qui différencie la capacité et le taux de germination au cours d'une période donnée. Pour V. nilotica, Yousif et al. (2020) ont montré que la couche épidermique du tissu palissadique du tégument des semences est dense, ce qui empêche l'imbibition des semences (Mensah et Ekeke, 2016). Le fort taux de germination obtenu chez F. albida au cours des 4 mois de suivi 
de la germination montre que les semences de cette espèce sont susceptibles de germer toutes dans les conditions pluviométriques de la zone d'étude (3 à 4 mois de pluie). Par contre, les faibles taux de germination observés au cours des 4 mois de suivi chez $V$. nilotica et $S$. obtusifolia montrent qu'il ne peut y avoir une germination presque complète de leurs semences dans les conditions climatiques de la zone d'étude. Ces semences non germées enrichissent le sol en semences (banque de semences de sol) et pourraient germer dans les années à venir si leur viabilité est conservée.

\section{Conclusion}

La capacité de dissémination des semences par les ruminants est liée au régime et à la préférence alimentaires propres à chaque animal, mais aussi à la phénologie des espèces végétales. Les fèces des caprins renferment plus de semences des espèces agroforestières (Vachellia nilotica, Balanites aegyptiaca, Faidherbia albida et Ziziphus mauritiana) tandis que les fèces des ovins contiennent celles de l'espèce invasive (Senna obtusifolia). Le passage des semences par le transit intestinal des animaux n'a pas permis d'améliorer de façon significative leurs taux de germination. L'endocarpe lignifié de $B$. aegyptiaca et de $Z$. mauritiana, les téguments durs des semences de $F$. albida, $P$. reticulatum, $S$. obtusifolia et $V$. nilotica constituent des barrières à la levée de la dormance. Même si le passage par le transit intestinal des animaux n’a pas amélioré significativement les taux de germination, la capacité à germer d'une portion des semences serait à l'origine de leur régénération dans les agrosystèmes dégradés où sont appliquées les fumures qui les contiennent. Les ovins (pour V. nilotica, B. aegyptiaca, F. albida et $S$. obtusifolia) et les bovins (pour Z. mauritiana et $F$. albida) offrent de meilleures possibilités de régénération au regard des taux de germination obtenus avec des délais et des durées de germination plus courts. Les animaux contribuent directement à modifier la diversité fonctionnelle des espèces dans les agroécosystèmes grâce à la dissémination des graines, et l'homme intervient indirectement par le parcage et l'apport des fèces stockées en enclos ou en fosses fumières en leur sein.

\section{Remerciements}

Les auteurs remercient le ministère de l'Enseignement supérieur et de la Recherche et de l'Innovation pour avoir octroyé une bourse d'étude au premier auteur et le CORAF-WECARD pour avoir soutenu cette étude à travers le projet d'amélioration de la résilience des agroécosystèmes (AmREACCAF)

\section{Références}

Abdou Habou M. K. H. R., Abdou L., Abdourahamane Illiassou S., Sanoussi Issoufou A. A. E., Soumana A., Mahamane A., 2019. Germination et croissance des plantules d'une espèce fruitière indigène au Niger : Balanites aegyptiaca (L.) Del. International Journal of Biological and Chemical Sciences, 13 (2): 693-703. https://dx.doi.org/10.4314/ijbcs.v13i2.10
Abdulazeez A., 2016. Effects of hot water on breaking seed dormancy of Senna obtusifolia from Bichi, Nigeria, in green house conditions. Journal of Agriculture and Veterinary Science, 9 (10): 29-32. https://doi.org/10.9790/2380-0910012932

Azad S., Manik M. R., Hasan S., Matin A., 2011. Effect of different pre-sowing treatments on seed germination percentage and growth performance of Acacia auriculiformis. Journal of Forestry Research, 22 (2): 183-188. https://doi.org/10.1007/s11676-011-0147-y

Bationo A. B., 1996. Étude de la régénération séminale des ligneux dans les jachères de Sobaka (Forêt classée de Nazinon, Burkina Faso). Mémoire DEA, Université de Ouagadougou, Burkina Faso, $54 \mathrm{p}$.

Bayen P., Traoré S., Bognounou F., Kaiser D., Thiombiano A., 2012. Effet du zaï amélioré sur la productivité du sorgho en zone sahélienne. Vertig0, 11 (3) : 1-10. http://id.erudit.org/ iderudit/1015054ar

Belem B., Nacoulma B. M. I., Gbangou R., Kambou S., Hansen H. H., Gausset Q., et al., 2007. Use of Non-Wood Forest Products by local people bordering the "Parc National Kaboré Tambi", Burkina Faso. The Journal of Transdisciplinary Environmental Studies, 6 (1): 1-21.

Bradford M. G., Westcott D. A., 2010. Consequences of southern cassowary (Casuarius casuarius L.) gut passage and deposition pattern on the germination of rainforest seeds. Austral Ecology, 35: 325-333. https://doi.org/10.1111/j.1442-9993.2009.02041.x

Burrows G. E., Virgona J. M., Heady R. D., 2009. Effect of boiling water, seed coat structure and provenance on the germination of Acacia melanoxylon seeds. Australian Journal of Botany, 57 (2): 139-147. https://doi.org/10.1071/BT08194

Centre Technique Forestier Tropical (Cirad-CTFT), 1988. Faidherbia albida (Del.) A. Chev. (synonyme : Acacia albida Del.). Monographie. Nogent-sur-Marne, France, Cirad-CTFT, 72 p. https://agritrop.cirad. fr/375602/

César J., Zoumana C., 1999. Les régimes alimentaires des bovins, ovins et caprins dans les savanes de Côte-d'Ivoire, et leurs effets sur la végétation. Fourrages, $159:$ 237-252.

Cornelissen J. H. C. A., Lavorel S. B., Garnier E. B., Díaz S. C., Buchmann N. D., Gurvich D. E. C., et al., 2003. A handbook of protocols for standardised and easy measurement of plant functional traits worldwide. Australian Journal of Botany, 51: 335380. http://www.publish.csiro.au/journals/ajb

Crosaz Y., 1995. Lutte contre l'érosion des sols en montagne méditerranéenne : connaissance du matériel végétal et quantification de son impact sur l'érosion. Thèse de doctorat, Université de droit, d'économie et des sciences d'Aix-Marseille, France, $229 \mathrm{p}$.

Danthu P., Ickowicz A., Friot D., Manga D., Sarr A., 1996. Effet du passage par le tractus digestif des ruminants domestiques sur la germination des graines de légumineuses ligneuses des zones tropicales sèches. Revue d'Élevage et de Médecine Vétérinaire des Pays Tropicaux, 49 (3) : 235-242. https://doi.org/10.19182/ remvt.9520

Depommier D., 1996. Production fruitière et devenir des semences de Faidherbia albida : la part des insectes spermatophages et du bétail dans la régénération de l'espèce. In : Peltier R. (éd.). Les parcs à faidherbia, Montpellier, France, Cirad-Forêt, 9-22. https:// agritrop.cirad.fr/388629/

Depommier D., Janodet E., Olivier R., 1992. Faidherbia albida parks and their influence on soils and crops at Watinoma, Burkina Faso. In: Vandenbeldt R. J. (ed.). Faidherbia albida in the West African Semi-Arid Tropics. Nairobi, Kenya, ICRAF, 111-115. https://agritrop. cirad.fr/465590/

Diallo I., Danthu P., Sambou B., Dione D., Goudiaby A., Poulsen K., 1996. Effects of different pretreatments on the germination of Faidherbia albida (Del.) A. Chev. International Tree Crops Journal, 9: 31-36. https://doi.org/10.1080/01435698.1996.9752957

Dumont B., 1996. Préférences et sélection alimentaire au pâturage. Productions Animales, 9 (5) : 359-365. 
Dumont B., Farruggia A., Garel J. P., 2007. Pâturage et biodiversité des prairies permanentes. Rencontres autour des Recherches sur les Ruminants, 14 (1) : 17-24.

Elfeel A. A., Hindi S. Z., 2014. Balanites aegyptiaca (L.) Del. var. aegyptiaca seed composition and variability among three different intra-specific sources. Life Science Journal, 11 (7): 160-166. http://www.lifesciencesite.com/lsj/life1107/020 2399 Olife110714_160_166.pdf

Gebreyesus G. M., 2017. Livestock herders perception on the causes and effects of Senna obtusifolia L. invasion in rangelands of Northern Ethiopia. African Journal of Agricultural Research, 12 (42): 3081-3087. https://doi.org/10.5897/AJAR2017.12505

Ginane C., Dumont B., Baumont R., Prache S., Fleurance G., Farruggia A., 2008. Comprendre le comportement alimentaire des herbivores au pâturage : intérêts pour l'élevage et l'environnement. Rencontres autour des Recherches sur les Ruminants, 15 : 315-322.

Guèye M., Samb P. I., Nongonierma A., 1999. Effets du tractus digestif de chèvres sur la germination de Ziziphus mauritiana Lam. Tropicultura, 16-17 (3) : 109-112.

Karimpour S., Davarynejad G. H., Rouhbakhsh H., Ardakani E., 2013. Data on scarification and stratification treatments on germination and seedling growth of Ziziphus Jujuba seeds. Advances in Environmental Biology, 7 (3): 501-505.

Kheloufi A., Mansouri L. M., 2017. Effet de l'acide sulfurique sur la germination d'un arbre fourrager Vachellia nilotica (L.) subsp. tomentosa. Livestock Research for Rural Development, 29 (2) : 11.

Mensah S. I., Ekeke C., 2016. Effects of different pretreatments and seed coat on dormancy and germination of seeds of Senna obtusifolia (L.) H. S. Irwin \& Barneby (Fabaceae). International Journal of Biology, 8 (2): 77-84. https://doi.org/10.5539/ijb. v8n $2 \mathrm{p} 77$

Niang-Diop F., Sambou B., Lykke A., 2011. Contraintes de régénération naturelle de Prosopis africana: facteurs affectant la germination des graines. International Journal of Biological and Chemical Sciences, 4 (5) : 1693-1705. https://doi.org/10.4314/ ijbcs.v4i5.65578

Norden N., Daws M. I., Antoine C., Gonzalez M. A., Garwood N. C., Chave J., 2009. The relationship between seed mass and mean time to germination for 1037 tree species across five tropical forests. Functional Ecology, 23: 203-210. https://doi.org/10.1111/i.13652435.2008.01477.x

Ouédraogo P., Bationo B. A., Sanou J., Traoré S., Barry S., Dayamba S. D., et al., 2017. Uses and vulnerability of ligneous species exploited by local population of northern Burkina Faso in their adaptation strategies to changing environments. Agriculture \& Food Security, 6 (15): 1-16. https://doi.org/10.1186/s40066-0170090-z

Ouédraogo S. J., Alexandre D. Y., 1996. Dynamique des parcs à Faidherbia albida : contraintes écologiques et économiques sur le terroir de Watinoma au Burkina Faso. In : Les parcs à Faidherbia albida. Cahiers scientifiques du Cirad-Forêt, 12 : 191-202.

Rutter S. M., 2010. Review: Grazing preferences in sheep and cattle: Implications for production, the environment and animal welfare. Canadian Journal of Animal Science, 90 (3): 285-293. https://doi. org/10.4141/cjas09119

Samuels I. A., Levey D. J., 2005. Effects of gut passage on seed germination: do experiments answer the questions they ask? Functional Ecology, 19: 365-368. https://doi.org/10.1111/i.13652435.2005.00973.x

Satti J. M., Kiran Kumar M., Sandeep B. V., Sudhakar P., 2016. Effect of chemical treatments on germination Acacia nilotica $\mathrm{L}$. of seeds. International Journal of Biotechnology. Photon, 115: 456-462.

Suárez F., Malo J. E., 1997. La dissémination endozoochore dans les pâturages méditerranéens et la transhumance. In : Fabre P., Duclos J.-C., Molénat G. (éds). Transhumance. Actes des journées euro-méditerranéennes de la transhumance. Saint-Martin de Crau, France, Maison de la Transhumance/Cheminements, 247-262.
Traoré L., Ouédraogo I., Ouédraogo A., Thiombiano A., 2011. Perceptions, usages et vulnérabilité des ressources végétales ligneuses dans le Sud-Ouest. International Journal of Biological and Chemical Sciences, 5 (1) : 258-278.

Traveset A., Robertson A. W., Rodriguez-Pérez J., 2007. A review on the role of endozoochory in seed germination. In: Dennis $A$. J., Schupp E. W., Green R. J., Wescott D. A. (eds). Seed dispersal: theory and its application in a changing world. Wallingford, UK, CABI, 78-103. https://doi.org/10.1079/9781845931650.0078

Yélémou B., Yaméogo G., Barro A., Taonda S., Hien V., 2013. La production de sorgho dans un parc à Piliostigma reticulatum en zone nord-soudanienne du Burkina Faso. Tropicultura, 31 (3) : 154162.

Yélémou B., Yaméogo G., Millogo/Rasolodimby J., Hien, V., 2007. Germination sexuée et dynamique de développement de Piliostigma reticulatum (D.C.) Hochst, une espèce agroforestière du Burkina Faso. Sécheresse, 18 (3) : 185-192.

Yousif A. M. I., Wang Y. R., Dali C., 2020. Seed dormancy overcoming and seed coat structure change in Leucaena leucocephala and Acacia nilotica. Forest Science and Technology, 16 (1): 18-25. https://doi.org/10.1080/21580103.2019.1700832

Zougmoré R., Zida Z., Kambou N. F., 2003. Role of nutrient amendments in the success of half-moon soil and water conservation practice in semiarid Burkina Faso. Soil and Tillage Research, 71 (2): 143-149. https://doi.org/10.1016/S0167. 1987(03)00050-3

Zwarg A., Schmidt M., Janßen T., Hahn K., Zizka G., 2012. Plant diversity, functional traits and soil conditions of grass savannas on lateritic crusts (bowé) in south eastern Burkina Faso. Flora et Vegetatio Sudano-Sambesica, 15: 15-24.

\begin{tabular}{|l|l|}
\hline $\begin{array}{l}\text { Ouédraogo et al. - Contribution des auteurs } \\
\text { Rôle du contributeur } \\
\text { Conceptualisation }\end{array}$ & $\begin{array}{l}\text { Noms des auteurs } \\
\text { P. Ouédraogo, S. Traoré, } \\
\text { B. A. Bationo }\end{array}$ \\
\hline $\begin{array}{l}\text { Gestion des données } \\
\text { P. Ouédraogo, S. Traoré, B. M. I. } \\
\text { Nacoulma, E. Daboue, B. A. Bationo }\end{array}$ \\
\hline $\begin{array}{l}\text { Analyse formelle } \\
\text { Méthodologie }\end{array}$ & $\begin{array}{l}\text { Pacoulma, Ouédraogo, S. Traoré, B. M. I. } \\
\text { Naboue, B. A. Bationo }\end{array}$ \\
\hline $\begin{array}{l}\text { Visualisation } \\
\text { B. Ouédraogo, S. Traoré, }\end{array}$ \\
\hline $\begin{array}{l}\text { Écriture - Préparation } \\
\text { de l'ébauche originale }\end{array}$ & $\begin{array}{l}\text { P. Ouédraogo, S. Traoré, B. M. I. } \\
\text { Nacoulma, E. Daboue, B. A. Bationo }\end{array}$ \\
\hline $\begin{array}{l}\text { Écriture - Révision } \\
\text { et édition }\end{array}$ & $\begin{array}{l}\text { P. Ouédraogo, S. Traoré, B. M. I. } \\
\text { Nacoulma, E. Daboue, B. A. Bationo }\end{array}$ \\
\hline
\end{tabular}

Bois et Forêts des Tropiques - Revue scientifique du Cirad -

(c) Bois et Forêts des Tropiques (c) Cirad

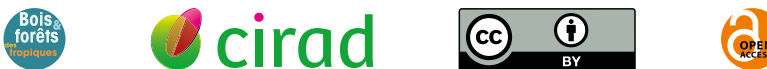

Cirad - Campus international de Baillarguet, 34398 Montpellier Cedex 5, France - Contact : bft@cirad.fr - ISSN : L-0006-579X 